\title{
ANÁLISE DA CONDUTIVIDADE ELÉTRICA, PH, SÓDIO E POTÁSSIO DA FOZ DO RIO PACOTI-CE
}

Felipe Nicolas de Morais Garcia - felipenicolasmg@ gmail.com

Instituto Federal do Ceará, IFCE

Andressa Guimarães Gomes - andressaguimaraes01 @ hotmail.com

Universidade de Fortaleza, UNIFOR 


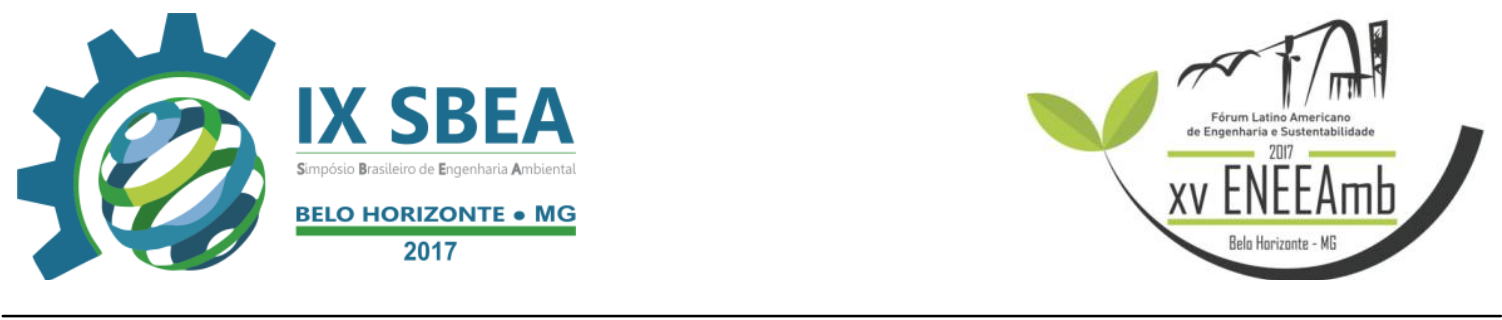

\section{RESUMO}

O presente artigo foi desenvolvido a partir de análises ex-situ realizadas com amostras de solo coletadas na foz do Rio Pacoti, um dos mais importantes rios da região metropolitana de Fortaleza localizado no estado do Ceará. Os parâmetros analisados foram o potencial hidrogeniônico, a condutividade elétrica, o sódio e o potássio

Palavras-chave: Analise de solos, pH, Sódio, Potassio, Condutividade Eletrica.

\section{INTRODUÇÃO/OBJETIVO}

O Rio Pacoti é o maior dos cursos d'água que atravessam a Região Metropolitana de Fortaleza, estando sua nascente na vertente-oriental da Serra de Baturité, percorrendo cerca de $150 \mathrm{~km}$ até desembocar no mar.

O Corredor Ecológico do Rio Pacoti, localizado no trecho compreendido entre a ponte velha da Rodovia CE 040 e a cota 600 (RN-IBGE) da Serra de Baturité, constituise em uma área que interliga duas áreas de proteção ambiental, a APA do Rio Pacoti e a APA da Serra de Baturité. No alto curso do Rio Pacoti observa-se a presença de vegetação de grande porte, composta por remanescentes da Mata Atlântica, já em seu médio curso constata-se a existência de espécies vegetais transicionais da floresta úmida para a caatinga, considerando que as formações florísticas variam de acordo com as condições de solo, clima, temperatura, umidade, topografia e influências antrópicas existentes em cada trecho.

A fauna, devido à grande variedade de ecossistemas é muito diversificada, com presença de répteis, anfíbios e mamíferos, além de várias espécies de aves e da fauna aquática. No Rio Pacoti encontram-se as barragens Pacoti e Riachão, que juntamente com a barragem do Gavião formam o principal complexo hídrico de abastecimento de água de Fortaleza (SEMACE,2010).

$\mathrm{O}$ objetivo da pesquisa foi analisar de uma forma mais precisa o Potencial Hidrogeniônico, a condutividade elétrica, o sódio e o potássio na foz do rio Pacoti, localizado no Ceará. 


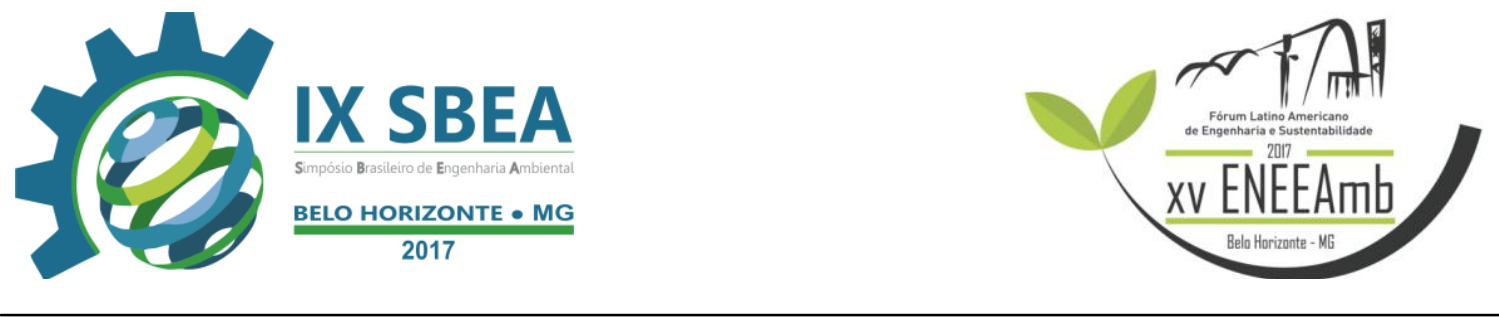

\section{METODOLOGIA}

A área de estudo foi a foz do rio Pacoti, localizado na divisa entre os municípios de Fortaleza e Aquiraz próximo a estrada da Cofeco como mostra a figura 1.

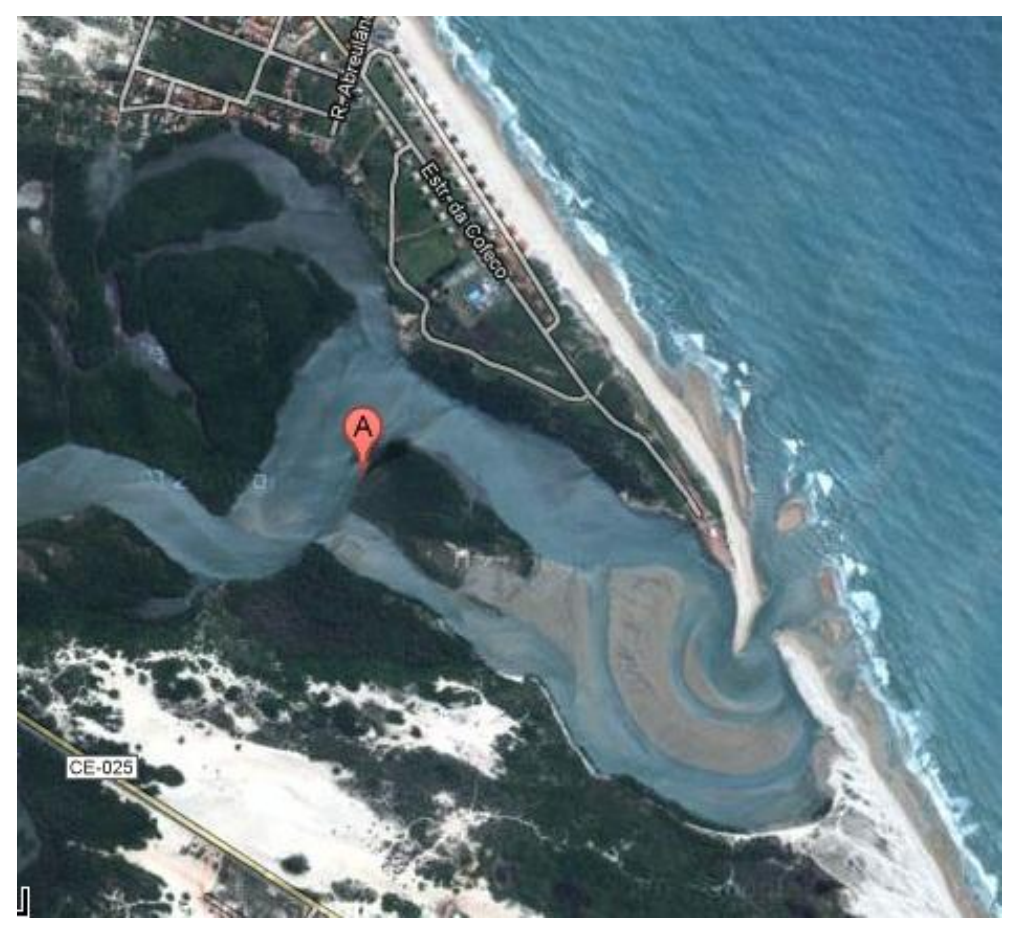

Figura 1: Local de realização da coleta

Fonte: Google Maps

A coleta realizou-se no dia 4 de agosto de 2012, onde período da estação era o seco. Foram coletadas amostras em diferentes pontos da Foz do Rio Pacoti, de acordo com a tabela 1 .

As amostras foram armazenadas em sacos plásticos, e em alguns pontos houve retirada de testemunho em canos de PVC de diferentes medidas de comprimento, mas com o mesmo diâmetro de $75 \mathrm{~mm}$. As amostras armazenadas em sacos plásticos foram acondicionadas em isopor, mantendo sua temperatura, até chegar ao Laboratório de Química Ambiental II (I-01) do Centro de Ciências Tecnológicas - CCT na Universidade de Fortaleza - UNIFOR. As amostras dos testemunhos foram mantidas 
em sentido horizontal para manter suas características até o momento de sua abertura e posterior análise.

Para os diferentes parâmetros analisados diferentes métodos foram utilizados, para potencial hidrogeniônico $(\mathrm{pH})$ as leituras foram feitas com o auxilio do pHmêtro, com o Método da Empresa Brasileira de Pesquisa Agropecuária - EMBRAPA,1997. Para a análise de condutividade elétrica (CE) utilizamos o aparelho condutivímetro (Analyser) método proposto pela EMBRAPA. Para Sódio (Na) e Potássio (K) utilizamos o equipamento Fotômetro de Chama (Analyser) com o método da EMBRAPA, 1997.

Tabela 1 - Metodologia Utilizada em Campo

\begin{tabular}{ll}
\hline Amostra & Metodologia \\
Mangue areia & Amostra de superfície \\
\hline Mangue & Testemunho e amostra de superfície \\
Estirâncio Superior & Amostra de superfície \\
\hline Estirâncio Médio & Amostra de superfície \\
Estirâncio Inferior & Amostra de superfície \\
Banco de Areia 01 e 02 & Testemunho \\
Ante - Praia & Amostra de superfície \\
Pós - Praia & Amostra de superfície \\
P7 Dentro & Amostra de superfície \\
P7 Fora & Amostra de superfície \\
P7 Margem & Testemunho e Amostra de superfície \\
\hline
\end{tabular}

\section{RESULTADOS E DISCUSSÃO}

As análises de $\mathrm{pH}$ foram realizadas em duplicata e os valores das médias se encontram na tabela 2. Em alguns pontos o $\mathrm{pH}$ foi classificado como extremamente ácido, muito ácido, ácido e pouco ácido, essa classificação pode ser vista na tabela 2 onde os limites de $\mathrm{pH}$ foram apresentados de acordo com Volkweiss (1989).

Os valores de condutividade elétrica também foram realizados em duplicada e depois foi realizada uma média para chega aos valores finais que se encontram na tabela 3. 
Tabela 2 - Classificação de pH de acordo com Volkweiss (1989).

\begin{tabular}{cc}
\hline pH(água) & Classificação \\
\hline$<5,0$ & Extremamente Ácido \\
$\mathbf{5 , 0}-\mathbf{5 , 5}$ & Muito ácido \\
$\mathbf{5 , 6}-\mathbf{6 , 0}$ & Ácido \\
$\mathbf{6 , 1}-\mathbf{6 , 5}$ & Pouco ácido \\
$\mathbf{6 , 6}-\mathbf{7 , 0}$ & Aproximadamente Neutro \\
$\mathbf{7 , 1}-\mathbf{7 , 5}$ & Pouco Alcalino (ou básico) \\
$\mathbf{7 , 6}-\mathbf{8 , 0}$ & Alcalino (ou básico) \\
$>\mathbf{8 , 0}$ & Muito Alcalino (ou básico) \\
\hline
\end{tabular}

Tabela 3 - Valores referentes a pH e CE

\begin{tabular}{lcc}
\hline Amostras & pH & C.E \\
\hline P7 Margem T/M 0 - 20 cm & 6,03 & $6,81 \mu \mathrm{S}$ \\
P7 Margem Meio 20 - 40 cm & 6,36 & 6,80 \\
P7 Margem M/B 40 - 61 cm & 5,94 & 5,76 \\
P7 Margem Base 61 - 78 cm & 6,72 & 2,58 \\
P7 Margem & 5,98 & 9,61 \\
P7 Dentro & 6,45 & 4,55 \\
P7 Fora & 6,58 & 4,41 \\
Mangue & 5,78 & 12,93 \\
Mangue Areia & 6,38 & 2,70 \\
Mangue Topo 0 - 15 cm & 4,45 & 15,68 \\
Mangue T/M 15 - 30 cm & 4,71 & 2,80 \\
Mangue Meio 30 - 45 cm & 4,68 & - \\
Mangue M/B 45 - 60 cm & 4,30 & 3,26 \\
Mangue Base 60 - 69,5 cm & 5,71 & 14,25 \\
Estirâncio Superior & 6,70 & 12,19 \\
Estirancio Médio & 6,54 & 3,11 \\
Estirancio Inferior & 5,24 & 5,38 \\
Pós - Praia & 6,35 & 1315 \\
Ante - Praia & 6,19 & 6,63 \\
Banco de Areia 01 Topo 0 & 6,55 & 2,37 \\
20cm & & 2,70 \\
Banco de Areia 01 T/M 20 - & 6,42 & \\
40cm & & 2,68 \\
Banco de Areia 01 M/B 40 - & 6,41 & 2,31 \\
60cm & & \\
Banco de Areia 01 Base 60 - & 6,05 & 73,7 \\
90 c & & 17,89 \\
Banco de Areia 02 Topo & 6,40 & \\
Banco de Areia 02 Meio & 6,47 & \\
\hline
\end{tabular}


Os valores de sódio e potássio, assim como os parâmetros citados anteriormente, foram realizados em duplicata e obtivemos a média desses valores chegando aos valores apresentados na tabela 4. Os limites de $\mathrm{k}+$, segundo o Centro de Pesquisa e Desenvolvimento de Solos e Recursos Ambientais foram apresentados na tabela 5.

Tabela 4 - valores referentes ao $\mathrm{Na}^{+}$e $\mathrm{K}^{+}$

\begin{tabular}{|c|c|c|}
\hline Amostra & $\mathrm{Na}^{+}$ & $\mathrm{K}^{+}$ \\
\hline P7 Margem T/M $0-20 \mathrm{~cm}$ & 38,00ppm & $5,1 \mathrm{ppm}$ \\
\hline P7 Margem Meio $20-40 \mathrm{~cm}$ & $52,18 \mathrm{ppm}$ & 8,5ppm \\
\hline P7 Margem M/B $40-61 \mathrm{~cm}$ & $30,50 \mathrm{ppm}$ & 3,55ppm \\
\hline P7 Margem Base $61-78 \mathrm{~cm}$ & $21,75 \mathrm{ppm}$ & $1,75 \mathrm{ppm}$ \\
\hline P7 Margem & - & - \\
\hline P7 Dentro & 38,65ppm & 4,50ppm \\
\hline P7 Fora & $31,15 \mathrm{ppm}$ & 3,85ppm \\
\hline Mangue & 33,35ppm & 4,85ppm \\
\hline Mangue Areia & $20,7 \mathrm{ppm}$ & $2,1 \mathrm{ppm}$ \\
\hline Mangue Topo $0-15 \mathrm{~cm}$ & 86,20ppm & 20,60ppm \\
\hline Mangue T/M $15-30 \mathrm{~cm}$ & $89,35 \mathrm{ppm}$ & $22,05 \mathrm{ppm}$ \\
\hline Mangue Meio $30-45 \mathrm{~cm}$ & $103,00 \mathrm{ppm}$ & $27,25 p p m$ \\
\hline Mangue M/B $45-60 \mathrm{~cm}$ & $81,25 p p m$ & $21,05 p p m$ \\
\hline Mangue Base $60-69,5 \mathrm{~cm}$ & $84,45 \mathrm{ppm}$ & $23,65 \mathrm{ppm}$ \\
\hline Estirâncio Superior & $19,85 p p m$ & $2,1 \mathrm{ppm}$ \\
\hline Estirâncio Médio & $35,25 \mathrm{ppm}$ & 3,65ppm \\
\hline Estirâncio Inferior & $23,8 \mathrm{ppm}$ & $2,1 \mathrm{ppm}$ \\
\hline Pós - Praia & 29,95ppm & $3,2 \mathrm{ppm}$ \\
\hline Ante - Praia & 36,60ppm & $2,6 \mathrm{ppm}$ \\
\hline $\begin{array}{l}\text { Banco de Areia } 01 \text { Topo } 0- \\
20 \mathrm{~cm}\end{array}$ & 29,75ppm & 3,50ppm \\
\hline $\begin{array}{l}\text { Banco de Areia } 01 \text { T/M } 20- \\
40 \mathrm{~cm}\end{array}$ & $18,00 \mathrm{ppm}$ & 1,55ppm \\
\hline $\begin{array}{l}\text { Banco de Areia } 01 \text { M/B } 40- \\
60 \mathrm{~cm}\end{array}$ & $30,45 p p m$ & $3,1 \mathrm{ppm}$ \\
\hline $\begin{array}{l}\text { Banco de Areia } 01 \text { Base } 60- \\
90 \mathrm{~cm}\end{array}$ & 22,7ppm & $1,9 \mathrm{ppm}$ \\
\hline $\begin{array}{l}\text { Banco de Areia } 02 \text { Topo } 0 \text { - } \\
\text { 30cm }\end{array}$ & 13,05ppm & 2,25ppm \\
\hline $\begin{array}{l}\text { Banco de Areia } 02 \text { Meio } 30 \text { - } \\
60 \mathrm{~cm}\end{array}$ & 25,30ppm & $2,4 \mathrm{ppm}$ \\
\hline $\begin{array}{l}\text { Banco de Areia } 02 \text { Base } 60- \\
90 \mathrm{~cm}\end{array}$ & $17,15 p p m$ & 1,15ppm \\
\hline
\end{tabular}




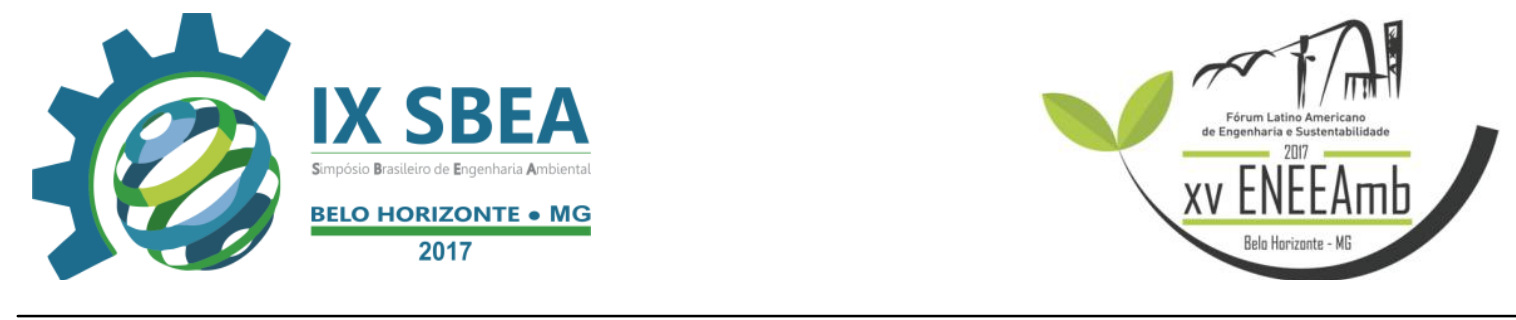

Tabela 5 - Limite de $\mathrm{k}^{+}$

\begin{tabular}{|c|c|c|c|c|c|c|}
\hline \multirow{2}{*}{ Teor } & \multirow{2}{*}{ Produção } & \multirow{2}{*}{$\mathrm{K}^{*}$ trocável } & \multicolumn{4}{|c|}{ Presina } \\
\hline & & & Florestais & Perenes & Anuais & Hortaliças \\
\hline & $\%$ & $\mathrm{mmol} / \mathrm{dm} \mathrm{m}^{3}$ & \multicolumn{4}{|c|}{${ }^{-} \mathrm{mg} / \mathrm{dm}^{3}$} \\
\hline Muito baixo & $0-70$ & $0,0-0,7$ & $0-2$ & $0-5$ & $0-6$ & $0-10$ \\
\hline Baixo & $71-90$ & $0,8-1,5$ & 3-5 & $6-12$ & $7-15$ & $11-25$ \\
\hline Médio & $91-100$ & $1,6-3,0$ & $6-8$ & $13-30$ & $16-40$ & $26 \cdot 60$ \\
\hline Alto & $>100$ & $3,1-6,0$ & 9-16 & $31-60$ & $41-80$ & $61-120$ \\
\hline Muito alto & $>100$ & $>6,0$ & $>16$ & $>60$ & $>80$ & $>120$ \\
\hline
\end{tabular}

Fonte: Centro de Pesquisa e Desenvolvimento de Solos e Recursos Ambientais

\section{CONCLUSÕES/RECOMENDAÇÕES}

As amostras mais ácidas são do mangue testemunho, e consequentemente os seus valores de condutividade elétrica são altos. A amostra mangue também é considerada ácida, pois está entre 5,6 - 6,0. A amostra mangue areia é considerada pouco ácida. Esse resultado já era esperado, pois o mangue é um ambiente halomórfico. Essas amostram apresentam altos valores de condutividade elétrica.

No P 7 testemunho, observa-se pontos ácidos, pouco ácidos e aproximadamente neutros. As amostras P7 margem, dentro e fora, são respectivamente ácidos, pouco ácido e pouco ácido. Nessas amostras, o P7 margem teve a condutividade elétrica alta. P7 dentro e P7 fora tiveram condutividades elétricas aproximadas. Nas amostras de estirâncios, a maior acidez foi no interior. No ante-praia e pós-praia tem pouca acidez, mas a condutividade do pós-praia foi o dobro do ante-praia. No testemunho do banco de areia, a maioria é pouco ácida, apenas uma amostra é considerada ácida; e a C.E foram baixíssimas nesses pontos. Nas amostras de banco de areia 02, todos os pontos são pouco ácidos, mas a condutividade elétrica tem valores totalmente diferentes.

Os valores de potássio são maiores nas amostras do mangue testemunho, concretizando a teoria. 


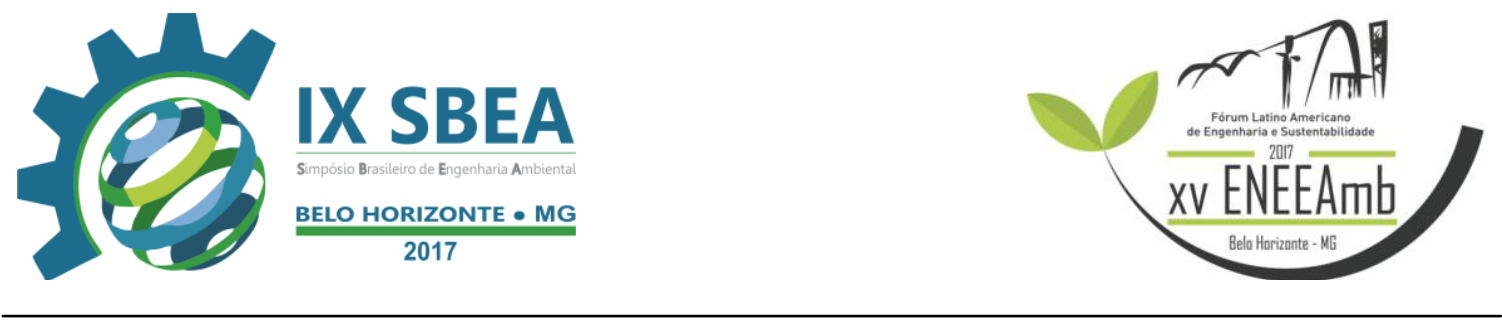

\section{REFERÊNCIAS BIBLIOGRÁFICAS}

EMBRAPA - Empresa Brasileira de Pesquisa Agropecuária. Sistema Brasileiro de Classificação de Solos. Brasília: Embrapa Produção de Informação, 1999. 412p.

EMBRAPA. Serviço Nacional de Levantamento e Conservação do Solo. Manual de métodos e análise de solo, 2a edição, Rio de Janeiro, 1997. 221 p.

Corredor Ecológico do rio Pacoti - Ceará. Disponível em: http://www.semace.ce.gov.br/2010/12/corredor-ecologico-do-rio-pacoti/. Acesso em: 27 de Nov..2012.

Instituto Brasileiro de Geografia e Estatística - IBGE. Manual Técnico de Pedologia. $4^{\circ}$ Ed. Rio de Janeiro: Coordenação de Recursos Naturais e Estudos Ambientais, 2007.316p.

LIEPSCH, I. F. 19 Lições de Pedologia. $1^{\text {o }}$.ed. São Paulo: Oficina de Textos, 2011.

Manual de Metodos de Analise do Solo. EMBRAPA. http://www.agencia.cnptia.embrapa.br/Repositorio/Manual+de+Metodos_000fzvhotqk0 2wx5ok0q43a0ram31wtr.pdf. Acesso em: 28 de Nov.. 2012

MIYAZAWA, M. et al. Efeito de material vegetal na acidez do solo. Revista Brasileira de Ciência do Solo, Campinas, v.17, n.3, p.411-416, 1993.

Volkweiss, S. J. Química da acidez do solo. In: Seminário sobre Corretivo da Acidez do Solo, 2, 1989, Santa Maria. Anais... Santa Maria: UFSM, 1989. p.3-7. 